\title{
Traduire
}

Ine autre perspective sur I t raduction

Revue française de la traduction

$242 \mid 2020$

Passons au vert

\section{La biodiversité : le parent pauvre du développement durable?}

\section{Marie-Agnès Schmitt}

\section{(2) OpenEdition}

1 Journals

Édition électronique

URL : http://journals.openedition.org/traduire/2011

DOI : 10.4000/traduire.2011

ISSN : 2272-9992

Éditeur

Société française des traducteurs

\section{Édition imprimée}

Date de publication : 30 juin 2020

Pagination : 55-67

ISSN : 0395-773X

\section{Référence électronique}

Marie-Agnès Schmitt, «La biodiversité : le parent pauvre du développement durable ? », Traduire [En ligne], 242 | 2020, mis en ligne le 15 juillet 2020, consulté le 20 décembre 2020. URL : http:// journals.openedition.org/traduire/2011 ; DOI : https://doi.org/10.4000/traduire.2011 


\section{La biodiversité :}

le parent pauvre du développement durable?

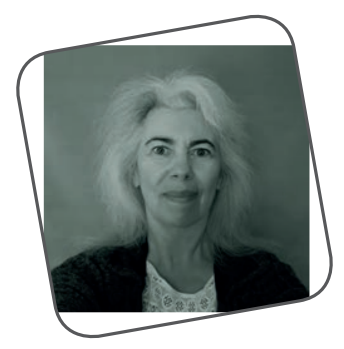

\section{Marie-Agnès Schmitt}

Finance et développement durable. Hier, deux mondes bien distincts aux motivations très différentes. Aujourd'hui, deux mondes qui se rejoignent pour œuvrer de concert sur la voie commune d'un avenir meilleur pour tous, au point que leurs terminologies respectives s'entremêlent. En vingtcing ans de métier, j'ai vu, et je vois encore, des termes du développement durable investir la sphère financière (on parle maintenant de "finance durable») et des termes techniques financiers s'inviter dans des documents consacrés au développement durable. Cette porosité de la frontière terminologique s'observe pour les documents grand public et les documents techniques. Cependant, la biodiversité, qui est pourtant un thème essentiel des objectifs de développement durable (ODD) définis par les États membres de l'Organisation des Nations unies (ONU), est moins touchée par ce mouvement que d'autres thèmes comme l'eau ou les énergies renouvelables. 
La biodiversité serait-elle donc le parent pauvre du développement durable? Cette question mériterait que l'on y consacre un livre entier. Je tenterai néanmoins d'apporter des éléments de réponse. Après avoir montré l'importance de la biodiversité pour l'être humain, j'analyserai les facteurs à l'origine de la porosité de la frontière terminologique pour expliquer la moindre présence de la biodiversité, puis j'étendrai l'analyse aux facteurs économiques, culturels et scientifiques, entre autres.

\section{Importance de la biodiversité}

La Commission mondiale sur l'environnement et le développement a défini en 1987 le développement durable comme un développement visant à "répondre aux besoins du présent sans compromettre la possibilité pour les générations à venir de satisfaire les leurs». Cette définition largement admise a été au cœur d'un travail de réflexion de longue haleine qui a donné lieu, entre autres, à l'adoption en 2015 de ce que l'on appelle les objectifs de développement durable: 17 ODD assortis de 169 cibles pour concrétiser ce «plan d'action pour l'humanité, la planète et la prospérité», qui couvre les trois dimensions du développement durable - économique, sociale et environnementale. La biodiversité est une préoccupation évoquée dans l'objectif 14.a - «[...] renforcer la contribution de la biodiversité marine au développement des pays en développement, en particulier des petits États insulaires en développement et des pays les moins avancés» - et l'objectif 15 - «[...] mettre fin à l'appauvrissement de la biodiversité».

Dans cette vision de l'avenir, un développement durable n'est donc pas envisageable sans biodiversité. Pourquoi? Parce que la biodiversité rend à l'être humain, directement ou indirectement, de nombreux services écosystémiques plus ou moins visibles. Par exemple, environ $30 \%$ de nos aliments dépendent de la pollinisation par des insectes pour pouvoir se reproduire. On pense bien sûr aux abeilles mellifères, mais la pollinisation est plus efficace, et les fruits et légumes ainsi obtenus plus nombreux, lorsque les insectes pollinisateurs sont sauvages et diversifiés. Autre exemple: les récifs coralliens protègent les côtes de l'érosion en amortissant la force des vagues et procurent directement nourriture 
et revenus (pêche, tourisme) à plus de 275 millions de personnes dans le monde.

Malgré ses bienfaits, la biodiversité terrestre et marine s'est considérablement appauvrie. Selon l'ONU, $50 \%$ des coraux de la planète ont péri ces trente dernières années. D'après la Plateforme intergouvernementale scientifique et politique sur la biodiversité et les services écosystémiques, plus d'un million d'espèces sont menacées d'extinction, dont un grand nombre dans les prochaines décennies, et le taux mondial actuel d'extinction est dix à cent fois supérieur au taux naturel et s'accélère. Selon le rapport 2019 du Forum économique mondial, le recul de la biodiversité fait partie des dix principaux risques en termes d'impact (au rang 6 sur 10) et en termes de probabilité de survenance (au rang 8 sur 10).

$\checkmark u$ l'importance vitale des enjeux, on pourrait s'attendre à ce que la biodiversité soit une priorité majeure de tout un chacun (citoyens, entreprises, associations, gouvernements à tous les niveaux) et à ce que cela se traduise par une montée en puissance du thème de la biodiversité tant dans les documents grand public que dans les documents techniques. Qu'en est-il?

\section{Une frontière terminologique poreuse}

\section{Un zeste de développement durable dans l'océan boursier}

Qui veut investir en Bourse peut placer son argent directement dans des titres (actions, obligations...) ou indirectement dans des fonds. Que l'on gère soi-même son portefeville ou que l'on en délègue la gestion, le choix des titres exige d'étudier les performances passées et actuelles des émetteurs pour estimer le futur retour sur investissement et le risque encouru. L'analyse financière permet, en examinant les comptes de l'entreprise, d'évaluer sa solvabilité, sa rentabilité et ses perspectives en termes absolus (coût de production, prix de vente, marge bénéficiaire, rapport cours/ bénéfices, ratio dettes/fonds propres, etc.) et en termes relatifs (situation de l'entreprise par rapport à ses concurrents, à la moyenne du secteur, dans la chaîne de valeur, etc.). Cependant, elle ne suffit pas, car une entreprise c'est plus que des chiffres: c'est une combinaison variable de processus 
organisationnels et décisionnels, de relations interpersonnelles et de rapports de force. Le jeu de ces éléments a des répercussions directes et indirectes au sein et à l'extérieur de l'entreprise.

La prise en compte d'aspects autres que financiers (risque d'atteinte à la réputation, modes de gestion du personnel, etc.) n'est pas un fait nouveau, car ils influent sur la performance de l'entreprise et donc sur le retour sur investissement. Ce qui est nouveau, c'est l'élargissement de la palette des aspects extra-financiers et la systématisation en cours de leur prise en compte. En effet, la prise de conscience de la nécessité d'un développement durable a induit des changements de mentalité, un renforcement de l'arsenal juridique et une modification des attentes des citoyens/clients. De plus en plus d'entreprises en ont tenu compte pour éviter d'éventuels conflits (grèves, boycotts, par exemple) et procès (pour polIution ou discrimination salariale, par exemple) susceptibles de ternir leur réputation et d'amputer leurs résultats financiers. Cela leur permet aussi d'être mieux placées que leurs concurrents qui n'ont pas suivi le mouvement et, finalement, de mieux vendre leurs produits et services.

Les analystes et les agences de notation ont intégré cette évolution d'ensemble et les efforts des entreprises dans l'analyse des performances de celles-ci. De leur côté, pour gérer efficacement les portefeuilles, les investisseurs en ont aussi tenu compte (dans leurs propres analyses ou en s'appuyant sur celles des spécialistes). Le phénomène a aussi conduit à la création de fonds à thématique environnementale, cotés en Bourse, à l'intention des investisseurs soucieux de l'environnement. Parmi ce type de fonds, rares sont ceux intégrant la biodiversité dans leurs critères extra-financiers, notamment parce que les entreprises se préoccupant de la biodiversité ou ayant un modèle économique directement fondé sur elle (par exemple, les parcs animaliers et floraux) sont peu nombreuses. Dans cette classe d'actifs, les grands thèmes sont l'eau, les énergies renouvelables, les déchets dans une perspective de développement durable - autrement dit, les fonds sont investis dans des entreprises qui servent directement l'être humain. Toutefois, ces critères environnementaux servent aussi indirectement la biodiversité, car l'assainissement et une gestion durable de l'eau, la production d'énergies 
renouvelables (moins émissives de $\mathrm{CO}_{2}$ ) et le recyclage des déchets atténuent les pressions sur la nature.

Ainsi, par le biais de la notion d'investissement socialement responsable (ISR) et des critères environnementaux, sociaux et de gouvernance (ESG) qui permettent d'évaluer la performance extra-financière de l'entreprise, la terminologie du développement durable a graduellement investi des documents comme les analyses/notations d'entreprises, les commentaires boursiers, les rapports de gestion de portefeuille ou de fonds et les rapports annuels d'entreprise. Cependant, la biodiversité est moins présente dans ces documents que d'autres thèmes environnementaux. En effet, dans la catégorie des critères environnementaux, si les termes «impact environnemental», "empreinte carbone» ou «recyclage» sont fréquents, les termes «biodiversité » ou «écosystèmes» le sont beaucoup moins.

\section{Une pincée d'instruments de marché dans le développement durable}

En 2014, la Conférence des Nations unies sur le commerce et le développement estimait le montant annuel des investissements requis pour atteindre les ODD à 5000-7000 milliards de dollars américains (USD) au niveau mondial et à 300-4500 milliards USD pour les seuls pays en développement. Selon la Convention sur la biodiversité (CBD), il faudrait entre 150 et 440 milliards USD par an jusqu'en 2020 pour réaliser son plan stratégique pour la biodiversité. Certes, ces chiffres sont des estimations, mais ils donnent un ordre de grandeur: les besoins sont colossaux (par comparaison, l'aide publique au développement a culminé à 132 milliards USD en 2015, selon l'Organisation de coopération et de développement économiques). Ce déficit de financement tient au grand nombre des ODD et des cibles, mais aussi au fait que les financeurs privés rechignent à investir dans ce type de projets, plus risqués et moins rentables.

La réalisation des ODD (biodiversité incluse) ne pouvant être financée uniquement sur fonds publics, il a fallu recourir à de nouvelles modalités de financement pour mobiliser des capitaux privés au service des ODD. Parmi ces modalités, on peut citer les partenariats public-privé (PPP) forgés dans 
cet objectif. Ce type de mécanisme de financement mixte permet de répondre aux besoins respectifs des diverses parties prenantes (financeurs publics, financeurs privés et demandeurs de fonds) en matière de prise de risques, d'objectif(s) et de retour sur investissement. Un PPP permet aux financeurs publics de contribuer à concrétiser des projets en plus grand nombre et de plus grande envergure, puisque les capitaux publics sont complétés par des capitaux privés, aux financeurs privés de prendre moins de risques, car ils bénéficient de la garantie apportée par les financeurs publics, et aux demandeurs de fonds d'accéder à des financements plus importants à un coût viable, car les financeurs publics abaissent le coût des fonds en leur assurant de meilleures conditions que celles du marché.

Ainsi, avec la multiplication des mécanismes de financement mixte de type PPP, les termes techniques comme «véhicule spécial» ou "entité ad hoc», "risque des premières pertes», «prise de participation», "prêt subordonné» se sont multipliés dans les documents relatifs au financement de projets de développement durable. Par exemple, au Pakistan, une entité ad hoc (Star Hydro Power) a été créée pour administrer le financement et la mise en œuvre du projet de construction d'une centrale hydroélectrique près de Patrind. Le cofinancement a été assuré sous forme de prêts accordés par des financeurs publics et de participations prises par des financeurs privés; l'Agence multilatérale de garantie des investissements (MIGA) s'est portée garante à concurrence de 148,5 millions USD.

Autre exemple d'évolution terminologique: I'apparition et la propagation du terme "obligation verte». Pour être qualifiées de vertes, les obligations doivent financer des projets dits «verts» et respecter les principes édictés par l'International Capital Market Association (ICMA). Le concept s'est répandu au point que le volume annuel mondial total des émissions est passé de 37 milliards USD en 2014 à 168 milliards USD en 2018 selon l'OCDE, mais la biodiversité est rarement le thème principal des obligations vertes. Elle peut toutefois en bénéficier indirectement. Par exemple, le produit de l'obligation verte (500 millions d'euros) émise en 2017 par la Caisse des Dépôts est affecté en partie à la restauration/dépollution de sites en vue de leur réutilisation, ce qui 
permet d'éviter de nouvelles destructions d'écosystèmes. Par ailleurs sont apparus récemment les «crédits verts», dont le coût est fonction de la réalisation d'objectifs sociaux et/ou environnementaux: le taux d'intérêt diminue si l'objectif est atteint. Ils se différencient des obligations vertes par le fait qu'ils peuvent aussi servir au financement général des activités de l'entreprise.

On le voit, la terminologie évolue avec les outils financiers mis au service des ODD. La porosité de la frontière terminologique entre le monde de la finance et celui du développement durable reflète une certaine convergence d'intérêts de ces deux mondes. Malgré tout, force est de constater que la biodiversité est dans un état alarmant.

\section{Comment en est-on arrivé là?}

Divers facteurs président, directement ou indirectement, à la réduction de la biodiversité. Par commodité, je les ai regroupés en grandes catégories, mais il faut savoir qu'ils interagissent entre eux et qu'ils sont plus ou moins récents et prédominants dans tel ou tel pays, ou dans telle ou telle région, en fonction de son histoire propre.

\section{Un développement débridé}

\section{a) Croissance démographique et développement éco-} nomique. La population mondiale avoisinait 950 millions de personnes en 1800, 1,6 milliard en 1900 et 7,6 milliards en 2018. Cette évolution démographique, les révolutions industrielles successives et l'élévation des niveaux de vie ont grandement élargi l'éventail des biens et services fournis, augmenté de façon exponentielle le volume des déchets produits par l'humanité tout en changeant profondément leur nature (par exemple, davantage de matières difficilement ou non biodégradables comme le plastique, qui, ingéré par les animaux, peut se retrouver dans la chaîne alimentaire jusqu'à l'être humain). La pollution de l'air, des sols et de l'eau sans précédent ainsi engendrée contribue sensiblement à réduire la biodiversité. 
b) Changement d'utilisation des sols. Sous l'effet conjoint de divers facteurs (notamment la croissance démographique, l'essor de l'industrie et de l'agro-industrie, les déplacements de population dus aux guerres et, plus récemment, au changement climatique), l'urbanisation n'a cessé de progresser: entre 1950 et 2018, la part de la population mondiale urbaine a bondi de 30 à $55 \%$ et elle devrait atteindre $66 \%$ en 2030. Or l'urbanisation entraîne une perte d'habitat, qui induit une perte de biodiversité. L'agriculture n'est pas en reste: entre 1980 et 2000 , environ 6 millions d'hectares de forêt ont été rayés de la carte en Asie du Sud-Est pour créer des plantations de palmiers à huile et environ 42 millions d'hectares en Amérique latine pour élever du bétail. L'activité minière a, elle aussi, détruit l'habitat de maintes espèces et pollué considérablement les sols et l'eau (par exemple, en extrayant l'or par cyanuration). Résultat: la destruction et la fragmentation des écosystèmes appauvrissent la biodiversité.

c) Surexploitation des ressources animales, végétales et minérales. L'être humain fore toujours plus profond, remodèle une surface toujours plus grande de la planète et puise toujours plus dans le capital naturel. La liste des ressources qu'il utilise s'est considérablement allongée ( $d u$ bois, elle s'est étendue au pétrole, au gaz, puis à l'uranium, au platine, au lithium, etc.). Entre 1950 et 2010, le stock de thon rouge du Nord a diminué de $80 \%$ en raison de la surpêche. La quantité de ressources extraites de la nature et les méthodes employées pour les exploiter portent atteinte à la biodiversité (par exemple, la pêche à l'explosif tue les poissons visés, mais aussi ce dont ils se nourrissent, et ravage leur habitat).

d) Changement climatique. Multiplication des phénomènes météorologiques extrêmes, élévation du niveau des mers et de la température moyenne à la surface du globe, fonte des glaciers, raréfaction de l'eau: les effets du changement climatique affectent la planète entière. Cela provoque une migration de la faune et de la flore, en quête de conditions climatiques plus favorables, qui perturbe les écosystèmes délaissés et ceux colonisés. 


\section{Une réaction tardive}

Vu l'ampleur des dégâts et l'importance des enjeux, on peut se demander pourquoi l'être humain n'a pas agi plus tôt pour protéger la biodiversité. Là aussi, les raisons sont multiples et interdépendantes. Il fallait notamment:

a) Avoir conscience du problème. De l'aube de l'humanité à nos jours, le lien entre une partie de la population et la nature s'est distendu sous l'effet de quatre grands facteurs qui sont apparus à des époques différentes, mais qui se cumulent aujourd'hui. D'abord, une partie de l'humanité a acquis une vision anthropocentrique du monde: la nature est à son service, il est normal de la solliciter sans guère se soucier de son état. Deuxièmement, de tout temps l'être humain a cherché à comprendre le monde dans lequel il évolue. À mesure de ses découvertes, il s'est détaché de la nature pour s'appuyer de plus en plus sur la science. Troisièmement, la minéralisation des villes ayant fait disparaître de nombreuses espèces végétales et animales du paysage urbain, bon nombre de citadins ont une vision édulcorée de la biodiversité. En ville, surtout dans les mégalopoles, la problématique de la biodiversité n'est pas une réalité aussi palpable que les effets du changement climatique tels que les ouragans ou les canicules. Il est donc plus difficile à une grande partie de la population d'avoir conscience du rôle crucial de la biodiversité, des pressions exercées sur elle et de l'intérêt de la préserver. Dernier point, pendant longtemps l'éventualité du problème n'a pas effleuré l'être humain, car il considérait qu'il n'y avait pas de limite à ce que la planète pouvait produire pour satisfaire ses besoins ni de limite au volume des déchets qu'elle pouvait absorber. Ces visions du monde n'ont pas incité à une démarche holistique qui intègre systématiquement la biodiversité (ni à la recherche de solutions fondées sur la nature, telles que l'agroécologie).

b) L'identifier avec précision. Cela exige de savoir à quel point et comment les activités humaines affectent la biodiversité. Selon la définition de la CBD, la biodiversité est la "variabilité des organismes vivants de toute origine...; cela comprend la diversité au sein des espèces et entre espèces ainsi que celle des écosystèmes». 
Or l'évolution des espèces et des écosystèmes est complexe à étudier, car elle s'opère à des échelles spatiales et temporelles variées et transcende les disciplines et les frontières. Il faut non seulement disposer de modèles (prospectifs) fiables, ce qui exige d'abondantes données et des séries chronologiques longues, cohérentes, comparables internationalement et finement ventilées, mais aussi harmoniser les méthodologies et les systèmes d'information au niveau national et international, certaines espèces migrant chaque année d'un pays ou d'un continent à l'autre. En outre, la biodiversité étant un champ de recherche relativement jeune, on manque de données pour évaluer pleinement les effets des interactions entre espèces sur son maintien, d'autant plus que l'urbanisation et la modification des pratiques agricoles ont entraîné une perte du savoir sur la biodiversité et que certains outils d'observation et d'analyse sont relativement récents (ordinateurs puissants et compacts, satellites, GPS, matériel sous-marin, etc.).

c) Disposer des outils adéquats pour y remédier. La biodiversité est à l'origine de biens communs (comme l'air) et de services communs (les coraux atténuent l'érosion des côtes). En appauvrissant la biodiversité, les activités humaines réduisent la quantité et la qualité de ces biens et services communs, créant ainsi des externalités négatives dans la mesure où ils n'ont pas de marché ni de propriétaire ou de prix. Ces externalités négatives n'étant pas ou peu prises en compte spontanément, les intégrer exige de définir des droits de propriété et d'attribuer une valeur monétaire à ces biens/services communs, ce qui est compliqué et nécessite l'intervention de l'État (via la fiscalité, la législation, la soft law). Ainsi, en France, la loi n²016-1087 du 8 août 2016 a inscrit dans le Code civil la notion de préjudice écologique avec la nature comme victime, en stipulant que la réparation doit s'effectuer en priorité en nature. Cependant, il ne suffit pas qu'un pays édicte des lois, car les entreprises peuvent délocaliser leur production vers les pays ayant une législation environnementale moins contraignante, et il existe par ailleurs des activités illicites transfrontalières (braconnage, orpaillage illégal, etc.). Par conséquent, il faut en outre 
une action concertée entre les pays, ce qui requiert un dialogue, des échanges d'informations, une coordination et les moyens financiers et humains nécessaires à cet effet, tout en évitant les doublons.

\section{Conclusion}

La moindre présence terminologique de la biodiversité par rapport à d'autres thèmes du développement durable reflète le moindre intérêt qui lui a longtemps été porté. Le modèle de développement économique d'une grande partie de l'humanité, conjugué à une certaine vision du monde, concourt à réduire considérablement la biodiversité. Cependant, les choses avancent. De plus en plus d'acteurs se mobilisent en faveur de la biodiversité et les initiatives se multiplient. Par exemple, des labels ont été créés (pêche durable et forêt durable, etc.), des végétaux sont réintroduits dans les villes pour que l'on puisse y bénéficier de leurs bienfaits (absorption du carbone, rafraîchissement, etc.), et la législation évolue. Un bémol cependant: attribuer une valeur monétaire aux biens et services communs fournis par la nature peut être une arme à double tranchant. Si cela permet d'attirer des capitaux privés, en complément des capitaux publics, pour préserver la nature, il faut néanmoins veiller à ce que cela ne vide pas cette démarche de son sens en réduisant aux yeux des gens la nature à un simple placement financier.

Le constat est manifeste: la biodiversité contribue à perpétuer le cycle de la vie et rend des services indispensables à l'humanité; la préserver exige de changer de paradigme, de repenser la relation être humain-nature et de ne pas apprécier la réussite uniquement à l'aune de critères économiques et financiers. Étudier la biodiversité, c'est étudier la dynamique de la vie sous ses multiples facettes, et c'est bien ce qui rend ce sujet si passionnant.

mas.traductions@orange.fr 
Marie-Agnès Schmitt est traductrice indépendante de I'anglais vers le français depuis 1995. Diplômée en économie et finance de l'université Paris Dauphine, elle s'intéresse depuis toujours à l'environnement. Ses domaines de spécialisation sont la finance, l'aide au développement et le développement durable. Elle traduit notamment pour des organisations internationales, de grandes banques et des sociétés d'analyse financière et se consacre également à la traduction d'articles de presse sur des sujets d'actualité.

\section{SOURCES CITÉES}

Ademe, Région Hauts-de-France, L'arbre en milieu urbain, acteur du climat en région Hauts-de-France, non daté.

Banque mondiale, https://www.banquemondiale.org/fr/news/immersive-story/ 2019/03/18/10-years-of-green-bonds-creating-the-blueprint-for-sustainabilityacross-capital-markets, consulté le 17/04/2020.

Caisse des Dépôts, Obligation verte, Rapport annuel 2018.

Commission mondiale pour l'environnement et le développement, Rapport de la Commission mondiale pour l'environnement et le développement, A/42/427, 4 août 1987.

Conférence des Nations unies sur le commerce et le développement, Rapport sur l'investissement dans le monde, L'investissement au service des objectifs de développement durable: un plan d'action, 2014.

European Union High-Level Expert Group on Sustainable Finance (HLEG), Financing a sustainable economy. Final Report 2018 by the High-Level Expert Group on Sustainable Finance.

Forum économique mondial, The Global Risks Report 2019, 14 $4^{\text {th }}$ Edition.

GARIBALDI Lucas A. et al., «Wild Pollinators Enhance Fruit Set of Crops Regardless of Honey Bee Abundance», in Science, vol. 339, 29 mars 2013.

Intergovernmental Panel on Climate Change (IPCC), 2019: IPCC Special Report on the Ocean and Cryosphere in a Changing Climate.

Investir, Les sociétés et les fonds qui s'engagent pour l'environnement, $\mathrm{n}^{\circ} 2410$, 14 mars 2020.

Le Monde diplomatique, https://www.monde-diplomatique.fr/cartes/1800-2050, consulté le 17/04/2020.

Organisation de coopération et de développement économiques (OCDE), Financer la biodiversité, agir pour l'économie et les entreprises, rapport préparé pour la réunion des ministres de l'Environnement du G7, les 5 et 6 mai 2019. 
Organisation de coopération et de développement économiques (OCDE), Coopération pour le développement 2016. Investir dans les objectifs de développement durable, choisir l'avenir, Paris, Éditions OCDE, 2016.

Organisation des Nations unies, Department of Economic and Social Affairs, Population Division, Population Facts, n²017/1, décembre 2018.

Organisation des Nations unies, Programme pour l'environnement, Le plastique marin: une nouvelle menace croissante pour les récifs coralliens, 5 mars 2018.

Organisation des Nations unies, Department of Economic and Social Affairs, Population Division, The World's Cities in 2018. Data Booklet.

Organisation des Nations unies, Transformer notre monde: le Programme de développement durable à l'horizon 2030, 18 septembre 2015.

Plateforme intergouvernementale scientifique et politique sur la biodiversité et les services écosystémiques, Résumé à l'intention des décideurs du Rapport sur l'évaluation mondiale de la biodiversité et des services écosystémiques de la Plateforme intergouvernementale scientifique et politique sur la biodiversité et les services écosystémiques, 29 mai 2019.

The Biodiversity Finance Initiative (BIOFIN), What is biodiversity finance? https:// www.biodiversityfinance.net/about-biofin/what-biodiversity-finance, consulté le $17 / 04 / 2020$.

World Wildlife Fund (WWF), Rapport Planète Vivante ${ }^{\circledR}$ 2018: Soyons ambitieux, WWF, Gland (Suisse).

World Wildlife Fund (WWF) en partenariat avec AXA, Into the wild, Intégrer la nature dans les stratégies d'investissement. Recommandations du WWF France et AXA pour les membres du G7 Environnement à Metz, les 5 et 6 mai 2019, octobre 2019. 